\title{
SUBSTANTIVISCHE DETERMINATIVKOMPOSITA IN DER JURISTISCHEN FACHSPRACHE UND IHRE ENTSPRECHUNGEN IM BOSNISCHEN
}

\section{EINLEITUNG}

Für die juristische Fachsprache ist unter anderem der Nominalstil charakteristisch. In diesem Beitrag werden die substantivischen Komposita in der deutschen juristischen Fachsprache untersucht. Die Komposita in der juristischen Terminologie sind oft sehr lang (Gesetzgebungszuständigkeit, Selbstverwaltungskörperschaft) und die unmittelbaren Konstituenten bestehen nicht selten aus mehreren Wörtern (Bundesgrenzschutzbehörde, Bundeshaushaltsgesetz). Das Untersuchungskorpus besteht aus 640 Beispielen aus dem Grundgesetz der BR Deutschland. Die Komposition ist in der bosnischen Sprache nicht das produktivste Wortbildungsmittel wie im Deutschen, deshalb ist es interessant zu erforschen, wie diese Komposita aus dem Deutschen ins Bosnische übersetzt werden. Das deutsche Grundgesetz wurde von einem Juristen ins Bosnische übersetzt. Diese Übersetzung war die Grundlage für die Ermittlung der Äquivalente in der Zielsprache. Da ich selbst Gerichtsdolmetscherin bin, habe ich auch meine eigene langjährige Erfahrung mit der Übersetzung von juristischen Texten sowohl in der Ausgangs- als auch in der Zielsprache miteinfließen lassen. Ergänzend dazu wurden für die Bestimmung der Äquivalente auch einsprachige sowie zweisprachige allgemeine sowie fachsprachliche Wörterbücher verwendet. Das Verwenden einer Übersetzung zu Analysezwecken war notwendig, weil bekanntlich nicht alle möglichen Komposita in den Wörterbüchern angeführt werden. Bevor die Ergebnisse der Korpusanalyse präsentiert werden, werden einige Fakten zum Thema Fachsprache und zu den Determinativkomposita allgemein präsentiert.

\section{ZUM THEMA FACHSPRACHE}

Heusinger (2004: 50) verwendet die Bezeichnung „Sonderlexik für die fachsprachliche Kommunikation“. Nach Heusinger (2004: 51) ist die Fachsprache eine Sondersprache

für eine eindeutige und widerspruchsfreie Kommunikation unter Fachleuten eines Fachgebietes, deren Funktionieren durch eine vereinbarte bzw. per definitionem festgelegte Terminologie und durch eine für die jeweilige Fachsprache charakteristische Grammatik wie auch Textstruktur entscheidend unterstützt wird.

* sanela.mesic@ff.unsa.ba 
Unter Termini versteht Heusinger (2004: 53)

im Rahmen einer Theorie begrifflich definierte Fachwörter, deren Invarianten (Seme, Bedeutungselemente) das Bezeichnete inhaltlich umgrenzen und gegenüber gemeinsprachlichen oder entlehnten formgleichen Lexemen wie kondensierte Texte zu begreifen sind, und sie sind in ihrer Funktion als Fachlexem folglich monosem (begrifflich eindeutig).

Manche gemeinsprachlichen Lexeme werden z. B. in der juristischen Fachsprache verwendet. Ihre fachspezifische Semantik wird dann in den Gesetzestexten definiert. So unterscheidet Schmidt (1969: 20, zitiert nach Heusinger 2004: 57) zwischen standardisierten und nicht standardisierten Termini. Die Definitionen eines standardisierten Terminus sind unter DIN (Deutsches Institut für Normierung e.V.) oder in Gesetzestexten zu finden (vgl. ebd.), während die nicht standardisierten Termini in Fachwörterbüchern lexikalisiert sind (vgl. Heusinger 2004: 62). Wenn es um die Form der Lexeme in den Fachsprachen geht, so ist laut Heusinger (2004: 62-63) Folgendes möglich: Beibehaltung der entlehnten Wörter, Wortbildung (als Beispiel wird ein Kompositum genannt), Wortgruppenbildung, Kurzwortbildung, künstliche Symbole, Formeln, Kunstwörter. Für diesen Aufsatz ist die Wortbildung interessant.

\section{SUBSTANTIVISCHE DETERMINATIVKOMPOSITA IM DEUTSCHEN}

Komposition ist die am häufigsten vorkommende Wortbildungsart im Deutschen. Ein Kompositum ist ein komplexes Wort und besteht ,aus anderen Wörtern (bzw. aus Morphemen)“ (Imo 2016: 7). Czichocki/Mrazović (1997: 249) definieren die Komposition als die „Bildung eines neuen Wortes aus mindestens zwei Grundmorphemen oder Morphemkonstruktionen“. Für Meibauer (2015: 32) ist die Komposition „die Bildung eines Wortes aus zwei (oder mehreren) vorhandenen Wörtern". Mit dem Aufbau dieser komplexen Wörter beschäftigt sich die Kompositionsmorphologie (vgl. Imo 2016: 7).

Elsen (2014) unterscheidet zwischen Determinativ-, Possessiv-, Kopulativ-, Reduplikativ- und verdeutlichenden Kompositionen. In der juristischen Fachsprache werden hauptsächlich substantivische Determinativkomposita verwendet. In der deutschen Gegenwartssprache ist das der Wortbildungstyp mit der höchsten Produktivität (vgl. Altmann 2011: 74).

Laut Altmann (ebd.) kann ein substantivisches Determinativkompositum aus folgenden Elementen bestehen: Nomen + Nomen (Betriebsrat), Adjektiv + Nomen (Altpapier), Verb + Nomen (Kaugummi), Adverb + Nomen (Sofort-Wirkung), Partikel + Nomen (Umfeld), Konfix + Nomen (Multivitamin). Bei anderen Autoren wie z. B. bei Czichocki/ Mrazović (1997: 48) werden jedoch teilweise andere Elemente genannt, so erscheinen bei diesen Autorinnen die Kombinationen Partikel + Nomen und Konfix + Nomen nicht, dafür aber Pronomen + Nomen (Ichform) und Numerale + Nomen (Zweikampf) (vgl. ebd.). Zu den Haupttypen der Nomen-Komposition zählen eindeutig die Nomen-Nomen-, Adjektiv-Nomen-, Verb-Nomen- und die Präposition-Nomen-Komposition (vgl. Meibauer 
et al. 2015: 48, Heringer 2013: 108). Die zuletzt genannte Kompositionsart kommt weniger häufig als die anderen vor (vgl. Eisenberg 1998: 217). Dieselben Typen werden auch bei Sahel/Vogel (2013: 94-95) angeführt.

Sehr detailliert ausgearbeitete semantische Typen der substantivischen Determinativkomposita sind bei Altmann (2011) zu finden. Altmann (2011: 77-78) unterscheidet bei den Nomen-Nomen-Komposita zwischen folgenden Bezeichnungstypen: 'affizierend' (Brotmaschine), 'effizierend' (Honigbiene), 'possessiv' (Hausherr), 'partitiv'/‘soziativ' (Vereinsmitglied), 'konstitutional' (Menschengruppe), 'substantiell'/'material' (Ledertasche), 'explikativ' (Verlustgeschäft), 'komparational' (Zitronenfalter), 'figurativ' (Würfelzucker), 'referentiell' (Schulangelegenheit), 'kompetentiell' (Verkehrsministerium), 'benefaktiv' (Hundefutter) und des Weiteren zwischen den sein-/tun-Prädikationen mit adverbialen Relationen: 'lokal' (Gartenlaube), 'direktional' (Nordwind), 'temporal' (Vorschulalter), 'instrumental' (Bleistiftskizze), 'konditional' (Notbremse), 'final' (Transportgebühr), 'konzessiv' (Pillenkinder), 'kausal' (Freudensprung) und 'modal' (Serienware).

Die semantischen Grundrelationen bei Nomen-Nomen-Komposita sind laut Fandrych/ Thurmair (1994, zit. nach Meibauer 2015: 54): SITUATION - ,ist in“ (Stadtautobahn), „,führt zu“(Gartentür), „,stammt aus/von“ (Erdöl), ,,ist zum Zeitpunkt/im Zeitraum“(Mittagessen); SITUATION-URHEBER - ,,ist verursacht von“ (Feuerschaden); KONSTITUTION - „,besteht ganz aus“ (Holztisch), ,hat" (Henkeltasse), ,,in der Art/Form/Farbe ... von" (Würfelzucker); KONSTITUTION-THEMA - „hat als Thema“ (Tierbuch), „im Bereich“ (Verkehrsministerium); ZWECK - „dient zu“ (Arbeitstisch), „schützt vor“ (Schmerztablette); INSTRUMENT - „funktioniert mit Hilfe von“(Benzinmotor).

Laut Zilić (2018: 21-22) kann das Verhältnis zwischen dem Bestimmungswort und dem Grundwort ein Zeitverhältnis (Septembertag), ein Ortsverhältnis (Wandtafel), ein Grundverhältnis (Liebeskummer), ein Zweckverhältnis (Wasserglas), ein Mittel- oder Werkzeugverhältnis (Windmühle), ein Stoffverhältnis (Papiergeld), ein Objektverhältnis (Tierzucht) oder ein attributives Verhältnis (Bergspitze) sein. Ebenso kann das Bestimmungswort den Stoff, mit dem sich die durch das Grundwort genannte Person beschäftigt, bezeichnen (Goldschmied), oder es handelt sich um einen Vergleich (Seidenhaar), eine Verstärkung (Hundekälte) oder um eine Verdeutlichung (Maultier) (vgl. ebd.).

Bei den Adjektiv-/Adverb/Numerale-Nomen-Komposita unterscheidet Altmann (2011: 78-79) zwischen folgenden Bezeichnungstypen: 'klassifikationsreflektierend' (Nur-Hausfrau), 'nur-modifikativ' (Gesamtmannschaft), 'explikativ' (Glatteis), 'temporal' (Sofort-Kredit), 'modal' (Universalprodukt), 'kausativ' (Vital-Tonikum), 'lokal' (Überall-Trend), 'direktional' (Rechtspfeil), 'indirekt agentiv' (Fremdherrschaft), 'indirekt affizierend' (Bronchialbonbon), 'indirekt effizierend' (Feinbäckerei), 'indirekt aktional' (Adoptivkind), 'indirekt lokal' (Schmalwand), 'indirekt direktional' (Ferntransport), 'indirekt temporal' (Frühnebel), 'indirekt modal' (Kolonialbauten), 'indirekt instrumental' (Grünfütterung), 'indirekt konstitutional' (Bronchialbäume), 'indirekt substantiell' (Kaltfront), 'indirekt partitiv' (Altstadt), 'indirekt possessiv' (Kolonialregime), 'indirekt substantiell' (Kriminalprozess). 
Weniger komplex ist die Einteilung der semantischen Muster bei Simoska (2012: 201-203). Diese Autorin unterscheidet zwischen semantischen Mustern, die keiner metaphorischen bzw. metonymischen Interpretation bedürfen, und denen, die dieser Interpretation bedürfen. $\mathrm{Zu}$ den ersten zählen das attributive (Glatteis), adjunktive (Simultanspiel), kausative (Bleichsucht) und indirekte Muster (Feinbäckerei) und zu den zweiten diejenigen Muster, die der metonymischen (Rothaut) und der metaphorischen Uminterpretation (Plumpsack) bedürfen (vgl. ebd.).

Bei den Verb-Nomen-Komposita gibt es laut Altmann (2011: 79) nur folgende Bezeichnungstypen: 'Agens' (Suchtrupp), 'affizierend' (Sendbote), 'effizierend' (Lachreiz), 'instrumental' (Rasierapparat), 'lokal'/'direktional' (Sendebereich), 'temporal' (Waschtag), 'konditional' (Abholpreis), 'kausal' (Lachfalten), 'substantiell' (Erzähltalent), 'modal' (Erzählstil) und 'explikativ' (Bastelarbeit). Diese Beziehungen zwischen den unmittelbaren Konstituenten eines Determinativkompositums sind für das Verständnis und ebenso für die Übersetzung in die Zielsprache wichtig.

\section{ERGEBNISSE DER KORPUSANALYSE}

Im Rahmen der empirischen Analyse wurden zuerst die substantivischen Determinativkomposita aus dem Grundgesetz ${ }^{1}$ analysiert und danach wurden ihre Entsprechungen im Bosnischen ermittelt.

\subsection{Substantivische Determinativkomposita im Grundgesetz der BR Deutschland}

Zunächst wurde die kategoriale Füllung der einzelnen Komposita aus dem Korpus untersucht, danach wurden die Bestandteile der unmittelbaren Konstituenten unter die Lupe genommen und zuletzt die Beziehungen zwischen den unmittelbaren Konstituenten beleuchtet. Für die Analyse waren die kategoriale Füllung und die semantische Relation zwischen den unmittelbaren Konstituenten der Komposita wichtig, weil von diesen beiden Elementen die Übersetzung ins Bosnische abhängt.

\subsubsection{Kategoriale Füllung und die Bestandteile der unmittelbaren Konstituenten}

Weiter oben wurde beschrieben, aus welchen Wortarten die substantivischen Komposita bestehen. Die Korpusanalyse hat Folgendes ergeben: Von insgesamt 631 substantivischen Determinativkomposita ${ }^{2}$ entfallen $569(90 \%)$ auf Nomen-Nomen-Komposita wie z. B. Abfallbeseitigung 3 , Bekenntnisschule, Bundeskanzler. An der zweiten Stelle sind Adjektiv-Nomen-Komposita (23 Beispiele): Disziplinarverfahren, Fernverkehr, Gesamtaufkommen. An der dritten Position sind Verb-Nomen-Komposita (21

1 Der gesamte Text des Grundgesetzes umfasst ca. 11330 Wörter. Viele Determinativkomposita wiederholen sich im Text.

2 An dieser Stelle ist anzumerken, dass alle Determinativkomposita im Grundgesetz analysiert wurden. Darunter sind auch gemeinsprachliche Determinativkomposita.

3 Alle deutschen Beispiele wurden aus dem Grundgesetz und alle Übersetzungsäquivalente von Šarčević (2005) übernommen. In seinem Buch sind sowohl das Grundgesetz als auch die Übersetzung des Autors ins Bosnische abgedruckt. 
Beispiele): Heilberuf, Lehrfach, Streitfall. An der vierten Position sind Präposition-Nomen-Komposita (9 Beispiele): Binnenschifffahrt, Unteroffizier, Vorjahr. An der fünften Stelle sind Adverb-Nomen-Komposita (7 Beispiele): Mehrausgabe, Mindereinnahme, Nichtzugehörigkeit. Und an der fünften Stelle sind Numerale-Nomen-Komposita mit nur zwei Beispielen: Drittstaat und Hundersatz.

Determinativkomposita sind binär strukturiert. Sie bestehen aus einem Bestimmungswort (Determinans) und einem Grundwort (Determinatum). Beide können aus einem oder aus mehreren Wörtern bestehen. Unter den Nomen-Nomen-Komposita wurden folgende Möglichkeiten ermittelt:

- Das Bestimmungswort und das Grundwort bestehen aus jeweils einem Wort: Abfallbeseitigung, Altersgrenze, Amtseid;

- das Bestimmungswort besteht aus einem Wort, das Grundwort aus zwei Wörtern: Bundesarbeitsgericht, Bundesfinanzbehörde;

- das Bestimmungswort besteht aus einem Wort, das Grundwort aus drei Wörtern: Schienenpersonennahverkehr;

- das Bestimmungswort besteht aus zwei Wörtern, das Grundwort aus einem: Bundeswehrverwaltung, Dienstherrenbefugnis, Dienstleistungspflicht;

- das Bestimmungswort besteht aus drei Wörtern, das Grundwort aus einem: Bundesgrenzschutzbehörde;

- das Bestimmungswort und das Grundwort bestehen jeweils aus zwei Wörtern: Hochseeschiffahrt, Krankenhauspflegesatz.

Präposition-Nomen-Komposita können folgendermaßen zusammengesetzt sein:

- Das Bestimmungswort und das Grundwort bestehen aus jeweils einem Wort: Unteroffizier, Vorjahr;

- das Bestimmungswort besteht aus einem Wort und das Grundwort aus zwei Wörtern: Binnenschifffahrt, Binnenwasserstraße.

Bei folgenden Komposita bestehen das Bestimmungswort und das Grundwort im gesamten Korpus aus jeweils einem Wort:

- Adjektiv-Nomen-Komposita: Disziplinarverfahren, Eigenverantwortung, Höchstbetrag;

- Verb-Nomen-Komposita: Erbinformation, Heilberuf, Lehrfreiheit;

- Adverb-Nomen-Komposita: Mehrbelastung, Mindereinnahme, Nichtzugehörigkeit;

- Numerale-Nomen-Komposita: Drittstaat, Hundertsatz.

\subsubsection{Beziehungen zwischen den unmittelbaren Konstituenten}

Wenn es um die Beziehungen zwischen den beiden unmittelbaren Konstituenten geht, wurden bei den Nomen-Nomen-Komposita folgende Bezeichnungstypen nach der Bedeutungskategorie der ergänzenden Verbprädikation nach Altmann (2011: 77-78) erwartet: 'explikativ', 'referentiell', 'kompetentiell', 'lokal', 'final' und folgende Bezeichnungstypen beispielsweise nicht: 'substantiell/material', 'komparational', 
'figurativ'. Altmann (2011:77) erklärt den Bezeichnungstyp 'explikativ' wie folgt: „Eine Gleichsetzung von zwei Größen wird bezeichnet: sein-Prädikation“. Dieser Beschreibung entspricht das Kompositum Mitgliedstaat (,Staat, der ein Mitglied ist'). Der Bezeichnungstyp 'referentiell' wird von Altmann (ebd.) folgendermaßen erläutert: „Ein Bezug zwischen zwei Größen wird ausgedrückt; Prädikation: betrifft, bezieht sich auf“ und der Bezeichnungstyp 'kompetentiell' wie folgt: „Eine Größe wird nach ihrem Zuständigkeitsbereich benannt; Prädikation: ist zuständig für" .

Im Korpus wurden Belege für beide Typen gefunden. Referentiell ist das Verhältnis in folgenden Komposita: Landesgesetz (,Gesetz, das das Land betrifft ${ }^{*}$ ), Landesgesetzgebung, Landesrecht. Kompetentiell ist das Verhältnis in folgenden Komposita: Landesfinanzbehörde, Landesjustizminister, Landesregierung (,Regierung, die für das Land zuständig ist ${ }^{6}$ ). Das lokale Verhältnis ist z. B. in den Komposita Grenzschutz (,Schutz, der sich an der Grenze befindet'), Hochseefischerei, Hochseeschifffahrt, das finale Verhältnis in Ausbildungsbeihilfe, Ausbildungsveranstaltung, Betäubungsmittel (,Mittel, (das man einnimmt,) damit man betäubt wird') zu erkennen. Das konditionale Verhältnis ist im folgenden Kompositum zu erkennen: Katastrophenhilfe (,Hilfe, die man im Falle einer Katastrophe bekommt/leistet $^{\circ}$ ). Ein Beispiel für das kausale Verhältnis ist Naturkatastrophe (,Katastrophe, die durch die Natur verursacht wird'). Zilić (2018: 22) schreibt von einem Objektverhältnis zwischen dem Bestimmungswort und dem Grundwort. Sehr viele Komposita aus dem Korpus weisen dieses Verhältnis zwischen den beiden unmittelbaren Konstituenten auf: Abfallbeseitigung (,der Abfall wird beseitigt'), Amtspflichtverletzung, Amtsübernahme.

Unter den Adjektiv-Nomen-Komposita im Korpus konnten Belege für den Bezeichnungstyp 'nur-modifikativ' nach Altmann (2011: 78) gefunden werden: Gesamtaufkommen (,das gesamte Aufkommen'), Höchstbetrag.

Die semantische Analyse der Komposita ist an dieser Stelle keineswegs ausgeschöpft und sollte nur an ein paar Beispielen verdeutlicht werden. Im Folgenden werden die Ergebnisse der Analyse der Äquivalente präsentiert.

\section{2 Äquivalente in der bosnischen Sprache}

Im Bosnischen ist die substantivische Komposition möglich, obwohl sie, wie weiter oben erwähnt, nicht das produktivste Wortbildungsmittel ist. In der bosnischen Grammatik wird zwischen nichtsuffixalen (ribolov ,Fischfang ${ }^{\circ}$ ) und suffixalen Nomenkomposita (posl-o-dav-ac ,Arbeitgeber) unterschieden (vgl. Jahić/Halilović/Palić 2000: 322). Zwischen den Teilen eines Kompositums im Bosnischen kann das Interfix -o(posl-o-dav-ac) oder das Interfix - $\varnothing$ - (rođendan ,Geburtstag) stehen. Bei den Komposita mit dem Interfix -o- ist die Basis des ersten Kompositumteiles entweder ein Nomen (basnopisac ,Fabeldichter), ein Adjektiv (niskogradnja ,Tiefbau), ein Numerale (prvoborac ,Vorkämpfer) oder ein Pronomen (samoodbrana ,Selbstverteidigung) (vgl. ebd.). Die Komposita mit dem Interfix $-\varnothing$ - haben als zweiten Teil entweder ein Lexem (rođendan), ein gebundenes lexikalisches Morphem (Zenicatrans ${ }^{4}$ ) oder sie haben als ersten Teil ein gebundenes lexikalisches Morphem (aerolinija ,Fluglinie) oder

4 ,Zenicatrans ist der Name eines Busunternehmens in der Stadt Zenica‘. 
beide Teile bestehen aus jeweils einem gebundenen lexikalischen Morphem (diskoteka ,Diskothek ) (vgl. Jahić/Halilović/Palić 2000: 323).

Trotz diesen Möglichkeiten ist es schwierig, Komposita aus der deutschen juristischen Fachsprache mit Komposita ins Bosnische zu übersetzten. Dies bestätigt auch die Korpusanalyse. Nur $17 \%$ der Komposita aus dem Korpus wurden mit einem Wort ins Bosnische übersetzt. Nur in etwa 40 \% dieser Fälle ist die Übersetzung m. E. gelungen. Hier werden ein paar gelungene Übersetzungsäquivalente angeführt: Bergbau - rudarstvo, Berichterstattung - izvještavanje, Geschäftsordnung - poslovnik. Unter diesen Äquivalenten sind sehr wenige Komposita: Geschäftsbereich - djelokrug, Nationalsozialismus - nacionalsocijalizam, Neubau - novogradnja. Der Übersetzer hat bei der Übersetzung oft das Bestimmungswort oder sogar das Grundwort weggelassen: Grundfreiheiten - *slobode, Mitgliedstaat - *država, Aufenthaltsrecht - *boravak, Berufswahl - *zanimanje. Wird bei der Übersetzung das Bestimmungswort ignoriert, ist die Übersetzung unvollständig. Wird bei der Übersetzung das Grundwort nicht beachtet, ist die Übersetzung falsch. Diese missglückten Übersetzungen zeigen, dass es nicht einfach ist, die Komposita zu übersetzen oder dass dem Übersetzer nicht alle Bestandteile eines Kompositums gleich wichtig erscheinen. Grundfreiheiten sind im Bosnischen osnovne slobode, ein Mitgliedstaat ist država članica, das Aufenthaltsrecht pravo boravka und die Berufswahl izbor zanimanja. Für jedes der genannten Komposita ist in der Zielsprache eine Zweiwortphrase notwendig. Mal sind es wie bei osnovne slobode ein Adjektiv und ein Nomen, mal ist es eine Nominalphrase mit dem Genitiv wie bei pravo boravka und izbor zanimanja und mal sind es zwei Nomen im selben Kasus wie bei država članica.

Sogar 60 \% der Komposita wurden auch von dem Übersetzer mit Zweiwortphrasen ins Bosnische übersetzt, wie z. B. Abfallbeseitigung - odstranjivanje otpada, Berufssoldat - profesionalni vojnik, Diskriminierungsverbot - zabrana diskriminacije. Das liegt an der binären Struktur der deutschen Komposita. Die zwei Wörter im Bosnischen stehen in verschiedenen syntaktischen Verhältnissen zueinander. Sie sind zwar immer attributiv, aber die Attribuierung erfolgt manchmal durch ein Adjektiv (Vorjahr - prethodna godina, Altersgrenze - starosna granica, Arbeitsfähigkeit - radna sposobnost) manchmal durch ein Genitivattribut (Amtsübernahme - preuzimanje dužnosti, Begnadigungsrecht - pravo pomilovanja, Besatzungskosten - troškovi okupacije) und in einem einzigen Fall im gesamten Korpus durch eine Apposition (Mitgliedstaat - država članica).

Der Rest von 23\% wurde mit längeren Phrasen ins Bosnische übersetzt. Gerade diese längeren Umschreibungen sind interessant für die Analyse. Dazu zählen z. B. Arbeitslosenversicherung - osiguranje za slučaj nezaposlenosti, Einfuhrumsatzsteuer - porez koji se plaća pri uvozu, Zeugnisverweigerungsrecht-pravo na uskraćivanje svjedočenja. Es handelt sich hierbei um ziemlich lange Komposita, deren Entsprechungen im zweisprachigen Wörterbuch von Hansen-Kokoruš et al. (2005) nicht alle genauso übersetzt wurden. Die Entsprechung für die Arbeitslosenversicherung ist gleich wie bei Šarčević (2005), die Einfuhrumsatzsteuer ist nicht im Wörterbuch verzeichnet und das Zeugnisverweigerungsrecht ist pravo odbijanja svjedočenja. Der Terminus Arbeitslosenversicherung

5 Da es kein entsprechendes deutsch-bosnisches Wörterbuch gibt, wurde das deutsch-kroatische Wörterbuch von Hansen-Kokoruš et al. (2005) verwendet. 
wird bei Köbler (2016: 27) wie folgt definiert: „Zwangsversicherung [...] für Arbeitnehmer gegen die wirtschaftlichen Folgen der Arbeitslosigkeit“. Auf der Webseite Duden online heißt es: ,gesetzlich geregelte Pflichtversicherung gegen Nachteile durch Arbeitslosigkeit“. Der Terminus Einfuhrumsatzsteuer ist bei Köbler nicht verzeichnet und laut Duden online ist Folgendes darunter zu verstehen: „Sonderform der Umsatzsteuer für aus dem Ausland bezogene Ware“. Der Übersetzer hat diesen Terminus als Steuer, die bei der Einfuhr bezahlt wird übersetzt. Insofern ist das nicht die entsprechende Übersetzung. Das Zeugnisverweigerungsrecht ist laut Köbler (2016: 514) „das Recht eines zu einem Rechtsstreit geladenen Zeugen, sich der grundsätzlich bestehenden Pflicht, als Zeuge eine Aussage zu machen, zu entziehen“ und laut Duden online „Recht der Zeugnisverweigerung". Die Übersetzung des Übersetzers ist daher akzeptabel.

Im Kapitel 4.1.1. wurden noch längere Komposita als Beispiele genannt: Bundesgrenzschutzbehörde, Schienenpersonennahverkehr, Krankenhauspflegesatz. Wie erwartet, sind diese Determinativkomposita weder auf der Webseite Duden online noch im Wörterbuch von Köbler (2016) verzeichnet. Als Übersetzer muss man versuchen, solche Komposita zuerst in der Ausgangssprache zu paraphrasieren: Die Bundesgrenzschutzbehörde ist ,eine Behörde des Bundesgrenzschutzes'; der Schienenpersonennahverkehr ist ,der Personennahverkehr mittels Schienen'; der Krankenhauspflegesatz ist, der Pflegesatz für die Pflege in einem Krankenhaus'. Die Lexeme Bundesgrenzschutz, Personennahverkehr und Pflegesatz sind in den einsprachigen Wörterbüchern verzeichnet. Diese Tatsache hilft bei der Zerlegung der Komposita und bei der Übersetzung. Der Übersetzer hat diese drei Termini wie folgt übersetzt: Bundesgrenzschutzbehörde - Savezna pogranična služba, Schienenpersonennahverkehr - šinski putnički saobraćaj, Krankenhauspflegesatz - dodatak za njegu u bolnicama. Die Entsprechung für den Terminus Bundesgrenzschutzbehörde ist richtig. Das kann in einem juristischen Wörterbuch überprüft werden. Der Bundesgrenzschutz ist laut Köbler (2016: 79) ,die in bundeseigener Verwaltung geführte polizeiähnliche Bundesbehörde zum polizeilichen Schutz (der Grenzen) des Bundesgebietes“. Das Lexem Schienenpersonennahverkehr wurde nicht vollständig übersetzt. Die genaue Entsprechung wäre šinski putnički lokalni saobraćaj. Hier wurde bei der Übersetzung ein Teil des Kompositums weggelassen. Die korrekte Entsprechung für das Lexem Krankenhauspflegesatz wäre dnevni troškovi za njegu u bolnici. Zu dieser Lösung kommt man, wenn man als Übersetzer ein allgemeines einsprachiges und ein zweisprachiges Wörterbuch benutzt und die unmittelbaren Konstituenten des Kompositums nachschlägt. An mehreren Beispielen wurde hier verdeutlicht, wie man mit Hilfe von einsprachigen und zweisprachigen Wörterbüchern zu den entsprechenden Übersetzungen kommt, die bei solchen langen Komposita ausnahmslos Umschreibungen sind. Einfacher ist es, wenn etwas auch in der Zielsprache bereits existiert, auch wenn es keine Einwortentsprechung gibt. Bei der Ermittlung der Äquivalente helfen auch Paralleltexte in der Zielsprache.

\section{FAZIT}

Die Terminologie in jeder Fachsprache sollte eindeutig sein. Diese Eindeutigkeit wird im Deutschen durch die sehr langen Komposita, deren unmittelbare Konstituenten in verschiedenen Beziehungen zueinanderstehen können, erschwert. Deshalb ist es sehr 
wichtig, die Komposita vor der Übersetzung in die Zielsprache in ihre Bestandteile zu zerlegen und das Verhältnis zwischen den Konstituenten festzustellen. Dieser Schritt ist vor allem dann notwendig, wenn man die Äquivalente nicht in den entsprechenden ein- und zweisprachigen allgemein- und fachsprachlichen Wörterbüchern findet. Des Weiteren helfen auch hier wie bei vielen anderen Übersetzungsschwierigkeiten Paralleltexte in der Zielsprache. Diese Untersuchung hat gezeigt, wie schwierig es ist, die Determinativkomposita im Deutschen richtig zu verstehen und die entsprechenden Äquivalente im Bosnischen zu finden. Für eine größere Untersuchung kann das Korpus mit Beispielen aus anderen juristischen Texten erweitert werden.

\section{Quellentext}

ŠARČEVIĆ, Edin (2005) Ustavno uređenje Savezne Republike Njemačke: Osnove njemačkog Državnog prava (sa izvornim tekstom Ustava i prijevodom na bosanski jezik). Sarajevo: KULT/B, Fondacija Heinrich Böll - Regionalni ured, Ambasada Savezne Republike Njemačke u Bosni i Hercegovini.

\section{Literatur}

ALTMANN, Hans (2011) Prüfungswissen Wortbildung. 3., durchgesehene Auflage. Göttingen: Vandenhoeck \& Ruprecht.

CZICHOCKI, Sieglinde/Pavica MRAZOVIĆ (1997) Lexikologie der deutschen Sprache: Einführung. Szeged: Jate.

Duden online. https://www.duden.de [15.01.2019].

EISENBERG, Peter (1998) Grundriß der deutschen Grammatik. Band 1: Das Wort. Stuttgart/Weimar: J. B. Metzler.

ELSEN, Hilke (2014) Grundzüge der Morphologie des Deutschen. Berlin/Boston: de Gruyter.

HANSEN-KOKORUŠ, Renata/Josip MATEŠIĆ/Zrinka PEČUR-MEDINGER/Marija ZNIKA (2005) Deutsch-kroatisches Universalwörterbuch. Zagreb: Globus.

HERINGER, Hans Jürgen (2013) Deutsche Grammatik: Ein Arbeitsbuch für Studierende und Lehrende. Paderborn: Wilhelm Fink.

HEUSINGER, Siegfried (2004) Die Lexik der deutschen Gegenwartssprache. München: Wilhelm Fink.

IMO, Wolfgang (2016) Grammatik. Eine Einführung. Stuttgart: Metzler.

JAHIĆ, Dževad/Senahid HALILOVIĆ/Ismail PALIĆ (2000) Gramatika bosanskoga jezika. Zenica: Dom štampe.

KÖBLER, Gerhard (2016) Juristisches Wörterbuch: Für Studium und Ausbildung. 16., neubearbeitete Auflage. München: Verlag Franz Vahlen.

MEIBAUER, Jörg/Ulrike DAMSKE/Jochen GEILFUSS-WOLFGANG/Jürgen PAFEL/Karl Heinz RAMERS/Monika ROTHWEILER/Markus STEINBACH (2015) Einführung in die germanistische Linguistik. 3., überarbeitete und aktualisierte Auflage. Stuttgart/Weimar: J. B. Metzler.

SAHEL, Said/Ralf VOGEL (2014) Einführung in die Morphologie des Deutschen. Darmstadt: WBG. 
SIMOSKA, Silvana (2012) Gesundfutter statt Schnellgericht. Neues zum Alten Kompositionsmodell der deutschen Sprache. Skopje: Universität „Hll. Kyrill und Method“. ZILIĆ, Erminka (2018) Wortbildung der deutschen Gegenwartssprache. Tešanj: Planjax komerc.

\title{
Zusammenfassung \\ SUBSTANTIVISCHE DETERMINATIVKOMPOSITA \\ IN DER JURISTISCHEN FACHSPRACHE UND IHRE ENTSPRECHUNGEN IM BOSNISCHEN
}

\begin{abstract}
Dieser Beitrag beschäftigt sich mit den substantivischen Determinativkomposita, die in der juristischen Fachsprache verwendet werden. Diese Komposita sind oft sehr lang und die unmittelbaren Konstituenten bestehen nicht selten aus mehreren Wörtern. Es wird untersucht, aus welchen unmittelbaren Konstituenten die Komposita im Grundgesetz der Bundesrepublik Deutschland bestehen und in welchem Verhältnis diese Konstituenten zueinanderstehen. Außerdem werden anhand der Übersetzung des deutschen Grundgesetzes und anhand verschiedener Wörterbücher die möglichen Äquivalente einiger Komposita in der bosnischen Sprache ermittelt. Die Übersetzungsäquivalente sind insofern interessant, als im Bosnischen die Komposition nicht die produktivste Wortbildungsart ist. Der Übersetzer muss zudem genau wissen, in welchem Verhältnis die unmittelbaren Konstituenten des deutschen Kompositums stehen, um das richtige Äquivalent zu ermitteln. Es ist nicht möglich, alle deutschen Komposita in den Wörterbüchern zu finden, was die Suche nach den Äquivalenten in jeder Zielsprache enorm erschwert. Diese Untersuchung soll also unter anderem zeigen, was man als Übersetzer über die substantivischen Determinativkomposita wissen sollte und wie man bei der Übersetzung von substantivischen Determinativkomposita ins Bosnische, aber auch in jede andere Zielsprache verfahren sollte.
\end{abstract}

Schlüsselwörter: Wortbildung, Determinativkomposita, juristische Terminologie, Fachsprache, Äquivalent

\section{Abstract \\ DETERMINATIVE COMPOUND NOUNS IN LEGAL GERMAN AND THEIR TRANSLATION EQUIVALENTS IN BOSNIAN}

This paper examines determinative compound nouns in legal German. These compounds are often of considerable length and comprise multi-word constituents. The research aims at identifying the components of the determinative compound nouns found in the Basic Law for the Federal Republic of Germany, as well as studying their relations within the compounds. The possible translation equivalents in Bosnian are to be determined for several compounds on the basis of existing translations of the German 
constitution and various appropriate dictionaries. These translation equivalents require our utmost attention, since compounding is not the most productive word-formation process in the Bosnian language. Furthermore, precise translation equivalents are determined on teh basis of the correct interpretation of grammatical relations within the compounds themselves, which is an additional skill any translator must possess in order to arrive at a correct solution. Finally, dictionaries do not have entries for every German compound of this sort, which additionally complicates the process of their translation into any target language. This research therefore emphasises the specific knowledge a translator should possess regarding determinative compound nouns and the ways their translation into Bosnian or any other target language should be conducted.

Keywords: word formation, determinative compound nouns, legal term, LSP, translation equivalent

\section{Povzetek \\ SAMOSTALNIŠKE ZLOŽENKE V NEMŠKEM PRAVNEM JEZIKU IN NJIHOVE USTREZNICE V BOSANSKEM JEZIKU}

Prispevek se ukvarja s samostalniškimi zloženkami, ki se uporabljajo v pravnem jeziku. Običajno gre za zelo dolge besede, katerih sestavine so neredko sestavljene iz več besed. Raziskava zasleduje vprašanje, iz katerih neposrednih sestavin sestojijo zloženke v Splošnem državljanskem zakoniku Zvezne republike Nemčije in kakšno je njihovo razmerje. Poleg tega želi raziskava na osnovi prevodov nemškega Državljanskega zakonika ter različnih slovarjev ugotoviti, katere so možne ustreznice nekaterih zloženk v bosanskem jeziku. Prevodne ustreznice so zanimive tudi zato, ker v bosanščini zlaganje ni najproduktivnejša besedotvorna vrsta. Prevajalec mora poleg tega natančno vedeti, v kakšnem razmerju so neposredne sestavine zloženke, da bi tako ugotovil pravo ustreznico. Ker vseh nemških zloženk v slovarjih ni mogoče najti, je v vsakem ciljnem jeziku ugotavljanje ustreznic močno oteženo. Raziskava želi bi med drugim pokazati, kaj bi prevajalci morali vedeti o samostalniških zloženkah in kako naj bi jih prevajali v bosanski, pa tudi katerikoli drug ciljni jezik.

Ključne besede: besedotvorje, samostalniške zloženke, pravna terminologija, strokovni jezik, ustreznica 